Agrotrópica 32(2): 105 - 110. 2020.

Centro de Pesquisas do Cacau, Ilhéus, Bahia, Brasil

\title{
OCORRÊNCIA DE Aleurocanthus woglumi Ashby (HEMIPTERA: ALEYRODIDAE) NO SUL DA BAHIA*
}

\author{
Juliane Damasceno de Carvalho Neves, Felipe Lopes Neves ${ }^{2}$, Maria Aparecida Leão \\ Bittencourt ${ }^{3}$ \\ ${ }^{1}$ Universidade Estadual de Santa Cruz (UESC). Programa de Pós-graduação em Produção Vegetal, \\ julianedamasceno@agronoma.eng.br. \\ ${ }^{2}$ Engenheiro Agrônomo. Instituto Capixaba de Pesquisa, Assistência Técnica e Extensão Rural (INCAPER), \\ felipe.neves@incaper.es.gov.br \\ ${ }^{3}$ Universidade Estadual de Santa Cruz (UESC).Departamento de Ciências Agrárias e Ambientais. malbitte@uesc.br. \\ Autor para correspondência: julianedamasceno@agronoma.eng.br \\ *Parte da Dissertação de Mestrado do primeiro autor.
}

Foram coletadas folhas de diferentes espécies botânicas em pomares diversificados no Sul da Bahia, com objetivo de detectar hospedeiros e nível de infestação de Aleurocanthus woglumi Ashby (Hemiptera: Aleyrodidae). Em laboratório, observou-se a quantidade de posturas/folha, de ovos/postura, de ninfas/folhas e de pupários/ folhas. Os dados de todos os hospedeiros foram analisados separadamente por município e agrupados para a análise em nível de região. Aleurocanthus woglumi foi detectada em sete diferentes espécies botânicas hospedeiras: três espécies de citros (laranjeira-pera - Citrus sinensis (L.) Osbeck, limoeiro - Citrus $\times$ limonia Osbeck, tangerineira - C. reticulata Blanco) - (Rutaceae); mangueira (Mangifera indica L.- Anacardiaceae); jambeiro-vermelho (Syzygium malaccense L.- Myrtaceae); cafeeiro (Coffea canephora L. - Rubiaceae) e murta (Murraya paniculata L. - Rutaceae). As folhas de laranjeira-pera foram as mais infestadas, com média de $42,7 \%$.

Palavras-chave: Mosca-negra-dos-citros, Citrus spp., laranja.

\section{Occurrence of Aleurocanthus woglumi Ashby (Hemiptera: Aleyrodidae) in}

Southern Bahia. In order to detect hosts and level of Aleurocanthus woglumi Ashby, the infestation was evaluated on leaves of different plant species in different orchards. The number of eggs / leaf, the number of eggs / posture, the number of nymphs / leaves and the number of pupae / leaves was made in the laboratory. Data from all the hosts were analyzed separately by municipality, and grouped for analysis at the level of the region. A. woglumi was detected in seven different hostsand: three species of citrus (orange-pear - Citrus sinensis (L.) Osbeck, lemon tree - Citrus $\times$ limonia Osbeck, mandarin - C. reticulata Blanco) - (Rutaceae); mango tree (Mangifera indica L.- Anacardiaceae); red tree (Syzygium malaccense L. - Myrtaceae); coffee tree (Coffea canephora L. Rubiaceae) and myrtle (Murraya paniculata L. - Rutaceae). The pear orange leaves were the most infested with $42.7 \%$.

Key words: Mosca-negra-dos-citrus, Citrus spp., orange. 


\section{Introdução}

A mosca-negra-dos-citros Aleurocanthus woglumi Ashby (Hemiptera: Aleyrodidae) é originária da Ásia e encontra-se disseminada em várias partes do mundo, sendo detectada no Brasil em 2001, no Estado do Pará (Oliveira, Silva e Návia, 2001; Pena, 2007; Silva et al., 2010; Silva et al., 2011; Vendramin, Pena e Silva, 2015; Lima, 2018; Castilhos, 2019; Mapa, 2020). Na Bahia a primeira ocorrência registrada foi em 2010, em viveiros, hortos e áreas urbanas nas cidades de Caravelas e Teixeira de Freitas, localizadas na região Extremo Sul do estado (Silva et al., 2010). Espécies de Citrus spp. - (Rutaceae), cajueiro (Anacardium occidentale L. - Anacardiaceae) e abacateiro (Persea americana Miller - Lauraceae) são hospedeiros primários, mas podem infestar mais de 300 espécies de plantas (Mapa, 2020).

A mosca-negra-do-citros é encontrada em várias regiões do país devido a sua grande capacidade de dispersão e adaptação às diversas condições climáticas. A mosca-negra possui tamanho entre $0,8 \mathrm{~mm}$ e $1,7 \mathrm{~mm}$, apresenta aparelho bucal tipo sugador labial tanto na fase jovem como na adulta e coloração escura. Seus ovos são branco-leitosos, tornando-se marrom escuro próximo à eclosão das ninfas, são colocados agregados em forma de espiral, com média de 25 ovos variando de 7 a 61 ovos, na parte abaxial do limbo foliar, sendo que cada fêmea coloca de duas a três espirais de ovos durante seu ciclo de vida com postura média de 100 ovos durante seu ciclo biológico. O período de incubação varia de 10 a 18 dias, com média de 15 dias e viabilidade de 65 a 95\%. O ciclo biológico da postura à fase adulta, em laranja-pêra, varia de 48,8 a 126,7 dias. (Cunha, 2003; Dowell et al., 1981; Dowell, Fitzpatrick e Howard, 1978; Eppo, 1997; Jordão e Silva, 2006; Lemos et al., 2006; Martínez, 1983; Martínez \& Angeles, 1973; Patel \& Patel, 2001; Pena et al., 2009; Maia, 2010; Rossato, 2007; Saldanha, 2016).

Tanto os adultos como as ninfas causam danos e injúrias às plantas na face abaxial foliar ao se alimentarem no floema, sugando a seiva. Os insetos eliminam uma secreção açucarada que causa o aparecimento de fungos saprófitos, formando a fumagina que diminui a quantidade de luz incidente sobre os órgãos clorofilados da planta, reduzindo a capacidade de fotossíntese. O fato de se posicionarem na parte abaxial da folha também interfere com as trocas gasosas feitas através dos estômatos. (Gonçalves, 2013). Raga et al. (2012) caracterizaram o comportamento de oviposição de A.woglumi em um pomar comercial de citros, e observaram um total de 16.125 posturas obtendo-se, 63 posturas/folha no municipio de Artur Nogueira-SP. Em laboratório, observou-se que a lima ácida Tahiti apresentou maior número de posturas/planta, e ovos/ planta variando entre 17,4 e 35,8 e 211 e 568, respectivamente (Pena et al., 2009). Em condições de casa de vegetação, as brotações de laranjeira e limoeiro apresentaram 153,6 e 134,6 ovos/broto, respectivamente; enquanto a tangerineira apresentou a média de 47,4 ovos/broto; a mangueira 1,2 ovos/broto; a goiabeira com 8,6 ovos/broto; enquanto que o cajueiro não apresentou oviposição da mosca-negra-dos-citros (Lopes et al., 2013). Farias et al. (2011) observaram a ocorrência de A. woglumi atacando o mogno africano (Khaya ivorensis King), em áreas de reflorestamento na Amazônia Oriental, atacando plantas jovens (um ano) e em plantas adultas (5 anos), sendo os ovos colocados em espiral, na face abaxial da folha e cada postura com 35 a 50 ovos.

O objetivo deste estudo foi detectar hospedeiros e nível de infestação da mosca-negra-dos-citros em espécies botânicas em pomares diversificados no Sul da Bahia.

\section{Material e Métodos}

No período de janeiro a junho de 2014, foi realizado um levantamento populacional da mosca-negra-doscitros em pomares diversificados, em sete municípios da região Sul do estado da Bahia. Foram realizadas amostragens em Buerarema (14 ${ }^{\circ} 57^{\prime} \mathrm{S}$ e $39^{\circ} 17^{\prime} \mathrm{W}$; $107 \mathrm{~m})$, Camamu (13⒌ $58^{\prime} \mathrm{S}$ e $\left.39^{\circ} 8^{\prime} \mathrm{W} ; 27 \mathrm{~m}\right)$, Ilhéus $\left(14^{\circ} 47^{\prime} \mathrm{S}\right.$ e $\left.39^{\circ} 02^{\prime} \mathrm{W} ; 52 \mathrm{~m}\right)$, Itabuna $\left(14^{\circ} 47^{\prime} \mathrm{S}\right.$ e $\left.39^{\circ} 16^{\prime} \mathrm{W} ; 54 \mathrm{~m}\right)$, Ituberá $\left(13^{\circ} 43^{\prime} \mathrm{S}\right.$ e $\left.39^{\circ} 08^{\prime} \mathrm{W} ; 27 \mathrm{~m}\right)$, Una ( $15^{\circ} 17^{\prime} \mathrm{S}$ e $39^{\circ} 04^{\prime} \mathrm{W}$; $28 \mathrm{~m}$ ) e Valença (13⒉'S e $39^{\circ} 04^{\prime} \mathrm{W} ; 39 \mathrm{~m}$ ), tendo sido realizada apenas uma coleta em cada município.

O clima da região Sul da Bahia é o tropical, com pouca variação da temperatura média anual de $24^{\circ} \mathrm{C}$ a $29^{\circ} \mathrm{C}$.

Nos pomares, as amostragens foram realizadas em possíveis espécies hospedeiras como: mamoeiro, 
goiabeira, cajueiro, roseiras, citros, murta, jambeirovermelho, mangueira e abacateiro. As plantas foram selecionadas ao acaso e observadas às brotações e a face abaxial das folhas novas, com auxílio de lupa de bolso (10x a 30x de aumento) à procura de sinais (posturas, ninfas, pupários e adultos) da mosca-negrados-citros. Em cada pomar, foram selecionadas 10 plantas e retiradas 40 folhas de cada planta. As folhas foram acondicionadas em sacos de papel e estes colocados dentro de sacos plásticos, etiquetados com a data da coleta, espécie vegetal hospedeira e o município da coleta.

As amostras coletadas foram transportadas para o laboratório de Controle Biológico da Universidade Estadual de Santa Cruz (UESC) para serem analisadas. A contagem do número de posturas por folha, do número de ovos por postura e de ninfas e de pupários por folha foi realizada sob microscópio estereoscópico (56x). Os dados de todos os hospedeiros foram analisados separadamente por município e posteriormente agrupados para a análise em nível de região.

\section{Resultados e Discussão}

A ocorrência de A. woglumi (ovos, ninfas e adultos) foi detectada na região Sul da Bahia nas suas diferentes fases (Figura 1). A mosca-negra-dos-citros foi detectada em sete diferentes espécies botânicas hospedeiras: três espécies de citros (laranjeira-pêra - Citrus sinensis (L.) Osbeck, limoeiro - Citrus $\times$ limonia Osbeck, tangerineira - C. reticulata Blanco) - (Rutaceae), mangueira (Mangifera indica L.- Anacardiaceae), jambeiro-vermelho (Syzygium malaccense L.Myrtaceae), cafeeiro (Coffea canephora L. Rubiaceae) e murta (Murraya paniculata L. Rutaceae) (Tabela 1), corroborando com dados da literatura (Cunha, 2003; Dowell, 1979; Eppo, 2017; Oliveira, Silva e Návia, 1999; Lopes et al., 2010; Monteiro et al., 2012; Mapa, 2020).

Não foi detectada a presença da mosca-negra-doscitros em abacateiro e cajueiro, considerados como hospedeiros primários, e nem em goiabeira e mamoeiro diferindo do registrado em outros estudos (Mapa, 2020; Monteiro et al., 2012; Pena et al., 2009).

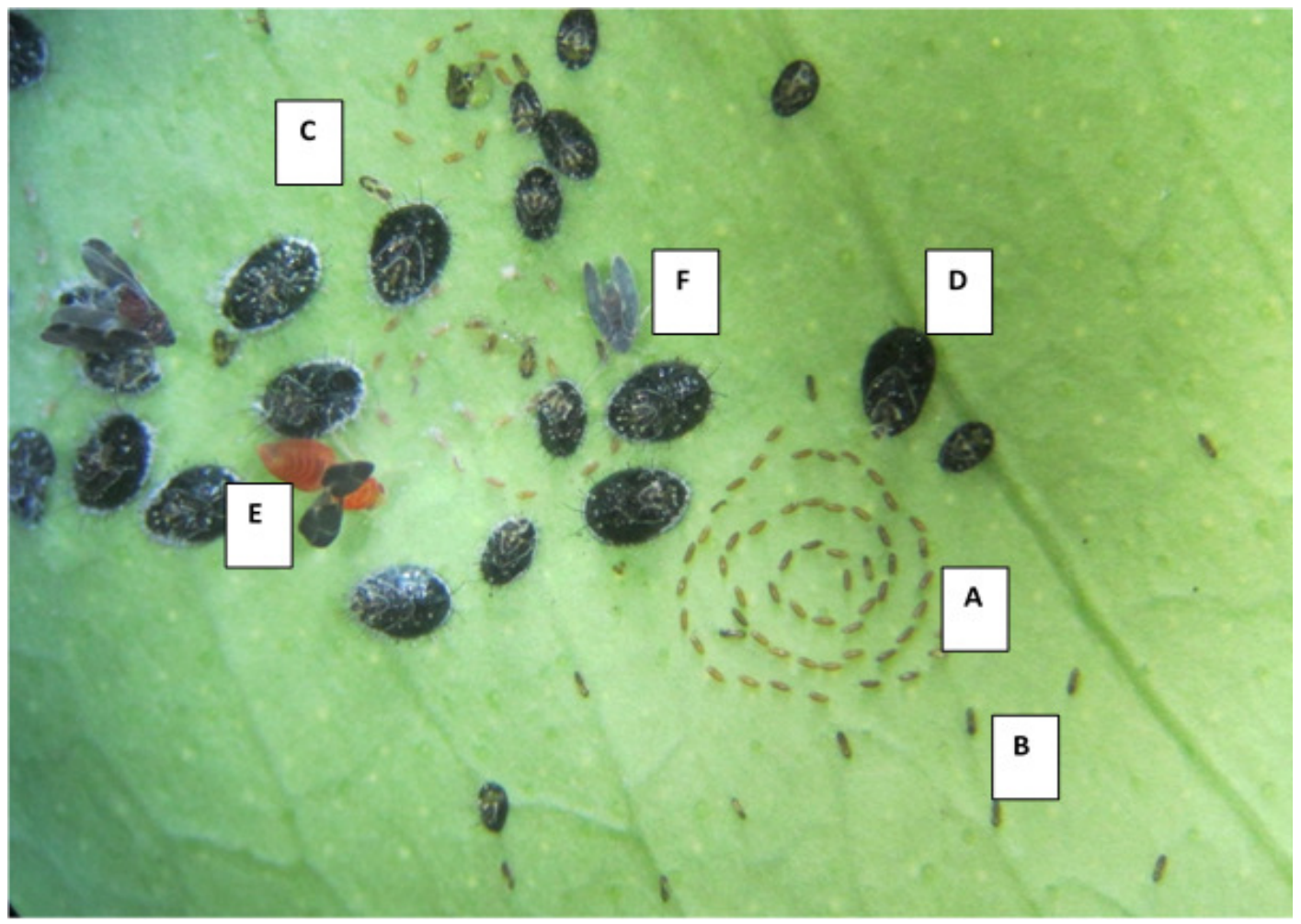

Figura 1 - Folha de laranjeira-pera com as diferentes fases de A. woglumi: ovos (A), ninfas (B, C), pupário (D), adulto recém emergido, (E) e adulto (F) (Fonte: Arquivo pessoal - coletada em Buerarema -Bahia). 
Tabela 1. Números de posturas/folha, ovos/postura, ninfas/postura e pupários/postura em hospedeiros nos diferentes municípios estudados

\begin{tabular}{|c|c|c|c|c|c|c|c|c|}
\hline \multirow{2}{*}{ Municípios } & \multirow{2}{*}{ Total } & \multicolumn{7}{|c|}{ Hospedeiros } \\
\hline & & Laranja-pera & Limão & Tangerina & Murta & Manga & Jambo vermelho & Café conilon \\
\hline \multirow{4}{*}{ Ilhéus } & Posturas/folha & 4,41 & 3,38 & 2,57 & 1,81 & 2,98 & 2,82 & 0 \\
\hline & Ovos/postura & 28,96 & 29,42 & 24,69 & 13,25 & 26,81 & 28,22 & 0 \\
\hline & Ninfas/postura & 2,21 & 1,79 & 0,88 & 1,49 & 1,21 & 0,82 & 0 \\
\hline & Pupários/postura & 1,28 & 1,11 & 1,95 & 2 & 0,89 & 1,12 & 0 \\
\hline \multirow{4}{*}{ Itabuna } & Posturas/folha & 3,97 & 3,81 & 2,47 & 0 & 3,45 & 2,44 & 0 \\
\hline & Ovos/postura & 29,36 & 29,72 & 24,43 & 0 & 27,74 & 25,32 & 0 \\
\hline & Ninfas/postura & 2,31 & 1,91 & 0,74 & 0 & 0,9 & 0,71 & 0 \\
\hline & Pupários/postura & 1,44 & 1 & 1,02 & 0 & 0,68 & 1,02 & 0 \\
\hline \multirow{4}{*}{ Buerarema } & Posturas/folha & 6,41 & 3,91 & 2,22 & 0 & 2,63 & 1,04 & 2,32 \\
\hline & Ovos/postura & 31,50 & 30,44 & 19,99 & 0 & 24,74 & 16,91 & 17,68 \\
\hline & Ninfas/postura & 2,81 & 1,79 & 1,22 & 0 & 1,06 & 0,52 & 0,45 \\
\hline & Pupários/postura & 6,69 & 4,83 & 2,99 & 0 & 1,59 & 0,69 & 0,93 \\
\hline \multirow{4}{*}{ Ituberá } & Posturas/folha & 2,57 & 2,4 & 0 & 0 & 0 & 0 & 0 \\
\hline & Ovos/postura & 23,82 & 23,54 & 0 & 0 & 0 & 0 & 0 \\
\hline & Ninfas/postura & 3,71 & 3,17 & 0 & 0 & 0 & 0 & 0 \\
\hline & Pupários/postura & 3,94 & 3,86 & 0 & 0 & 0 & 0 & 0 \\
\hline \multirow{4}{*}{ Camamu } & Posturas/folha & 2,18 & 2,3 & 0 & 0 & 0 & 0 & 0 \\
\hline & Ovos/postura & 20,03 & 21,03 & 0 & 0 & 0 & 0 & 0 \\
\hline & Ninfas/postura & 1,24 & 1,9 & 0 & 0 & 0 & 0 & 0 \\
\hline & Pupários/postura & 2,66 & 1,88 & 0 & 0 & 0 & 0 & 0 \\
\hline \multirow{4}{*}{ Valença } & Posturas/folha & 1,81 & 1,16 & 0 & 0 & 0 & 0 & 0 \\
\hline & Ovos/postura & 25,74 & 19,38 & 0 & 0 & 0 & 0 & 0 \\
\hline & Ninfas/postura & 1,68 & 2,29 & 0 & 0 & 0 & 0 & 0 \\
\hline & Pupários/postura & 1,92 & 1,76 & 0 & 0 & 0 & 0 & 0 \\
\hline \multirow{4}{*}{ una } & Posturas/folha & 3,17 & 2,93 & 0 & 0 & 2,89 & 0 & 0,35 \\
\hline & Ovos/postura & 24,42 & 20,61 & 0 & 0 & 20,48 & 0 & 16,17 \\
\hline & Ninfas/postura & 1,14 & 1,3 & 0 & 0 & 0,78 & 0 & 1,41 \\
\hline & Pupários/postura & 1,28 & 1,29 & 0 & 0 & 2,35 & 0 & 1,25 \\
\hline
\end{tabular}

Em cada pomar, foram selecionadas 10 plantas e retiradas 40 folhas de cada planta.

Em todos os municípios amostrados, observou-se que as plantas cítricas apresentaram maiores infestações em relação às outras espécies hospedeiras, correspondendo a $72 \%$ de folhas infestadas, corroborado por outros estudos que relataram os citros como hospedeiros preferenciais (Alvim, 2016; Cunha, 2003; Dowell, 1979; Eppo, 2017; Maia, 2010; Raga e Costa, 2008; Pena et al., 2009; Raga et al., 2012; Ronchi-Teles et al., 2009). Os citros apresentaram as maiores infestações nas folhas, sendo 42,7\% em laranjeira-pera, 39,3\% em limoeiro, e $16,0 \%$ na tangerineira.

Na mangueira, considerada hospedeiro primário, observou-se alta infestação $(19,8 \%)$ em relação aos outros hospedeiros não cítricos, semelhante ao relatado por outros autores (Maia, 2010; Pena et al., 2009; Raga et al., 2012).

O cafeeiro-conilon apresentou baixa a ocorrência da mosca-negra-dos-citros, tendo sido detectada apenas em Buerarema e Una, semelhante ao observado em Manaus (Ronchi-Teles et al., 2009). Em plantas de murta a ocorrência da mosca-negra-doscitros foi detectada apenas no município de Ilhéus.

Na região Sul da Bahia, o número de posturas por folhas de citros variou de 2,31 a 4,16 sendo estes resultados inferiores aos obtidos por Lemos et al. (2006) no estado do Maranhão, já o número de ovos por postura variou de 13,25 a 30,31 semelhante aos 
dados encontrados em outros estados brasileiros (Moraes et al., 2013; Farias et al., 2011; Lemos et al., 2006; Raga et al., 2012).

O número de ninfas da mosca-negra-dos-citros observados em folhas dos hospedeiros na região Sul da Bahia, variou de 1,07 a 18,12 ninfas/folhas.

Na região Sul da Bahia verificou-se a ocorrência da mosca-negra-dos-citros em apenas sete hospedeiros. O clima da região Sul da Bahia é favorável ao desenvolvimento do inseto como já foi observado em outros estudos, porém, nessa região o índice pluviométrico é elevado o que pode ter dificultado o desenvolvimento da praga em uma maior diversidade de hospedeiros (Moraes et al., 2013).

\section{Conclusões}

A mosca-negra-dos-citros ocorre na região Sul da Bahia, sendo que a Citrus sinensis apresenta as maiores infestações.

\section{Agradecimentos}

A Fundação de Amparo a Pesquisa do Estado da Bahia (FAPESB) pela concessão da bolsa, a Universidade Estadual de Santa Cruz (UESC) pela oportunidade do mestrado em Produção Vegetal e a todos que de alguma forma contribuíram para a realização desse trabalho.

\section{Literatura Citada}

ALVIM, R. G. 2016. Dissemination of Aleurocanthus woglumi in citrus plants, its natural enemies and new host plants in the state of Rio de Janeiro, Brazil. Ciência Rural 46(11):1891-1897.

CASTILHOS, R. V. 2019. Primeiro registro de Aleurocanthus woglumi (Hemiptera: Aleyrodidae) no estado de Santa Catarina, Brasil. Citrus Research Technology (Brasil) 40:1051.

CUNHA, M. L. A. 2003. Distribuição, hospedeiros, densidade populacional, aspectos biológicos e controle químico da mosca-negra-dos-citros (Aleurocanthus woglumi Ashby) nas condições do Estado do Pará. Dissertação Mestrado. Universidade Federal Rural da Amazônia, Manaus, AM. 54p.
DOWELL, R. et al. 1981. Biology plant insect relations and control of the citrus blackfly. Gainesville, Agricultural Experimental Station. Boletim técnico 818. pp.1-48.

DOWELL, R. V. 1979. Host selection by the citrus blackfly Aleurocanthus woglumi (Homoptera: Aleyrodidae). Entomologia experimentalis et appllicata 25(1):289-296.

DOWELL, R. V.; FITZPATRICK, G. E.; HOWRAD, F. W. 1978. Activity and dispersal of first instar larvae of the citrus blackfly. Journal of the New York Entomology Society 86(1):121-12

EUROPEAN AND MEDITERRANEAN PLANT PROTECTION ORGANIZATION - EPPO. 1997. Aleurocanthus woglumi. In: Eppo. Quarantine Pests for Europe, 2. ed, Wallingford, CAB International. pp.25-29.

EUROPEAN AND MEDITERRANEAN PLANT PROTECTION ORGANIZATION - EPPO. 2017. Disponível em: https://gd.eppo.int/taxon/ ALECWO/hosts.

FARIAS, P. R. S. et al. 2011. Ocorrência de Aleurocanthus woglumi em área de reflorestamento com mogno africano na Amazônia Oriental. Revista Ciência Agrária 54(1):85-88.

GONÇALVES, M. S. 2013. Flutuação populacional da mosca-negra-dos-citros, Aleurocanthus woglumi (Hemiptera: Aleyrodidae) e de seus inimigos naturais em plantios de citros. Dissertação Mestrado. Manaus, INPA. 68p.

JORDÃO, A. L.; SILVA, R. A. 2006. Guia de pragas agrícolas para o manejo integrado no Estado do Amapá. Riberão Preto, Holos. 182p.

LEMOS, R. N. S. et al. 2006. Ocorrência de Aleurocanthus woglumi Ashby (Hemiptera: Aleyrodidae) no Maranhão. Neotropical Entomology 35(4):558-559.

LIMA, B. M. F. V. et al. 2018. Entomopathogenic fungi associated with citrus blackfly (Aleurocanthus woglumi Ashby) in Southern Bahia. Arquivos do Instituto Biológico (online) 84:1-4.

LOPES, E. B. et al. 2010. Ocorrência da mosca-negrados-citros (Aleurocanthus woglumi) na Paraíba. Tecnologia \& Ciência Agropecuária 4(1):19-22. 
LOPES, G. S. et al. 2013. Preferência para oviposição e ciclo de vida de mosca-negra-dos-citros Aleurocanthus woglumi Ashby em espécies frutíferas. Revista Brasileira de Fruticultura 35(3):738-745.

MAIA, W. J. M. S. 2010. Manual de identificação de Aleurocathus woglumi, e seus inimigos naturais. $56 \mathrm{p}$.

MAPA. Cultura dos Citrus. 2020. Disponível em: $<$ http://www.agricultura.gov.br/vegetal/culturas/ citrus. pdf. $>$. Acesso em: 23 julho de 2020.

MARTÍNEZ, N. B.; ANGELES, N. 1973. Contribución al conocimiento de la biologia de la mosca prieta de los cítricos Aleurocanthus woglumi Ashby em Venezuela. Agronomía Tropical 23(1):401-406.

MARTÍNEZ, N. B. 1983. Biologia de la mosca prieta de los cítricos Aleurocanthus woglumi Ashby (Homoptera: Aleyrodidadae) em el campo. Agronomía Tropical 3(1):211-218.

MONTEIRO, B. S. et al. 2012. Ocorrência da moscanegra-dos-citros (Aleurocanthus woglumi Ashby) (Hemiptera: Aleyrodidae) em Pernambuco. Revista Caatinga 25(2):173-176.

MORAES, B. C. et al. 2013. Dinâmica bioclimática da Mosca-negra-dos-citrus no Brasil. Revista Brasileira de Climatologia 13(9):51-59.

OLIVEIRA, M. R. V.; SILVA, C. C. A.; NAVIA, D. 1999. Praga Quarentenária A1: a mosca negra dos citros, Aleurocanthus woglumi Ashby (Hemiptera: Aleyrodidae). EMBRAPA, Comunicado Técnico, 40.7p.

OLIVEIRA, M. R. V.; SILVIA, C. C. A; NAVIA, D. 2001. Mosca negra dos citros Aleurocanthus woglumi: alerta quarentenário. Ministério da Agricultura, Pecuária e Abastecimento, Brasília, DF. 12p.

PATEL, P. S.; PATEL, G. M., 2001. Biology of the citrus blackfly Aleurocanthus woglumi Ashby (Homoptera: Aleyrodidae) on Kagzi lime. Pest Management and Ecomonic Zoology 9(2):147-50.

PENA, M. R. et al. 2009. Biologia da mosca-negra-doscitros, Aleurocanthus woglumi Ashby (Hemiptera: Aleyrodidae), em três plantas hospedeiras. Neotropical Entomology 38(2):254-261.
PENA, M. R. 2007. Biologia da mosca-negra-doscitros, Aleurocanthus woglumi Ashby 1915 (Hemiptera:Aleyrodidae) em três plantas hospedeiras e uso do fungo Aschersonia sp., como agente entomopatogênico. Dissertação Mestrado. Universidade Federal do Amazonas, Manaus, AM. 97p.

RAGA, A. et al. 2012. Comportamento de oviposição da mosca-negra-dos-citros Aleurocanthus woglumi (Hemiptera: Aleyrodidae) em plantas cítricas. IDESIA 30(2):111-114.

RAGA, A.; COSTA, V. A. 2008. Mosca negra dos citros. Instituto Biológico, São Paulo, SP. Documento Técnico 001.9p.

RONCHI-TELES, B. et al. 2009. Observações sobre a ocorrência de mosca-negra-dos-citros, Aleurocanthus woglumi Ashby, 1915 (Hemiptera: Sternorrhyncha: Aleyrodidae) no Estado do Amazonas. Acta Amazônica 39(1):240-244.

ROSSATO, V. 2007. Ocorrência de Parasitóides de Aleurocanthus woglumi Ashby, 1903 (Hemiptera: Aleyrodidae) e seu Parasitismo por Cales noacki Howard, 1907 (Hymenoptera: Aphelinidae) nos Municípios de Belém, Capitão Poço e Irituia no Estado do Pará. Dissertação Mestrado. UFRA, Belém. 39p.

SALDANHA, C. B. 2016. Aleurocanthus woglumi ashby (Hemiptera: Aleyrodidae): flutuação populacional, distribuição espacial e levantamento de inimigos naturais. Dissertação Mestrado. São Paulo, SP. 51p.

SILVA, S. X. B. et al. 2010. Mosca-negra-dos-citros (Aleurocanthus woglumi Ashy) na Bahia: detecção e medidas de controle. In: Reunião Regional da SBPC no Recôncavo da Bahia.

SILVA, A. G. et al. 2011. Infestação da mosca-negrados-citros em pomares de citros em sistema de plantio convencional e agroflorestal. Revista Brasileira de Fruticultura 33(1):53-60.

VENDRAMIM, J. D.; PENA, M. R.; SILVA, N. M. 2015. Mosca-negra-dos-citros, Aleurocanthus woglumi Ashby. In: Vilela, E. F.; Zucchi, R. A. Pragas introduzidas no Brasil. Insetos e Ácaros. Piracicaba, SP, Fealq. pp.345-357. 